\title{
Assessment of stress adequacy with adenosine: Does the answer lie in the spleen?
}

\author{
William E. Moody, MRCP, PhD, a and Parthiban Arumugam, MBBS, FRCP \\ a Department of Cardiology, Centre for Clinical Cardiovascular Science, Nuffield House, Queen \\ Elizabeth Hospital Birmingham, University Hospital Birmingham NHS Foundation Trust, \\ Edgbaston, UK \\ b Department of Nuclear Medicine, Manchester Royal Infirmary, Manchester University NHS \\ Foundation Trust, Manchester, UK
}

Received Dec 5, 2020; accepted Dec 7, 2020

doi: $10.1007 /$ s12350-020-02485-7

\section{See related article, pp. 1205-1214}

Functional imaging plays an important part in the diagnosis and risk stratification of patients with known or suspected coronary artery disease (CAD). Results from an ASNC member survey performed in 2011 showed that pharmacological stress was used in well over half $(59 \%)$ of all radionuclide myocardial perfusion imaging (MPI) studies. ${ }^{1}$ Adenosine and regadenoson are commonly used pharmacological stress agents in both MPI studies and cardiovascular magnetic resonance (CMR) for the assessment of ischemia in CAD patients. As recommended by guidelines, the agents are either administered using a weight-based dosage over several minutes in the case of adenosine or a fixed dosage bolus independent of weight for regadenoson. ${ }^{2}$ The assumption is that if caffeine and antagonistic medications are withdrawn, the stress test is considered valid if administered as per guidelines. Unlike in exercise treadmill testing and dobutamine stress, there is no target heart rate endpoint to confirm the adequacy of stress with vasodilator stress. Although symptoms (chest pain and dyspnea) and ECG changes are non-specific in the context of vasodilator stress, most imaging centers will appropriately document patient-reported symptoms and hemodynamic response to help inform stress adequacy.

Reprint requests: Parthiban Arumugam, MBBS, FRCP, Department of Nuclear Medicine, Manchester Royal Infirmary, Manchester University NHS Foundation Trust, Oxford Road, ManchesterM13 9WL; Parthiban.Arumugam@cmft.nhs.uk

J Nucl Cardiol 2022;29:1215-8.

$1071-3581 / \$ 34.00$

Copyright (C) 2021 American Society of Nuclear Cardiology.
An adequate vasodilator response with adenosine infusion has been defined as an increase in heart rate of more than 10 beats per minute or a decrease in systolic blood pressure of at least $10 \mathrm{mmHg}^{3}$ Using these thresholds, up to a third of false-negative stress CMR results in the CE-MARC study were attributed to inadequate pharmacological vasodilator stress. ${ }^{4}$ The notion, however, that these hemodynamic cut-offs provide a reliable assessment of stress adequacy is misguided. Chronotropic incompetence is common in patients with cardiovascular disease (particularly that which involves the right coronary artery) and is itself an independent predictor of major adverse cardiovascular events and overall mortality. ${ }^{5}$ Patients with end-stage liver disease undergoing pre-transplant risk stratification may also be less suitable for pharmacological vasodilator stress because of high resting myocardial blood flows, ${ }^{6}$ a factor which likely contributes to the lower specificity and sensitivity of myocardial perfusion imaging in this disease cohort. ${ }^{7}$ Moreover, although recommended by guidelines, a number of commonly used cardiovascular medications (including beta-blockers, digitalis, calcium antagonists and amiodarone) are not uniformly withdrawn and could confound the heart rate response to stress. It is conceivable that these limitations in assessing stress adequacy contribute to the $10 \%$ rate of falsenegative PET MPI results, even when performed in expert centers. ${ }^{8}$ Collectively, these observations make it imperative for imaging physicians to use everything in their technological armamentarium to facilitate an objective assessment of stress adequacy and include this information in their reports as has been suggested in consensus guidelines. ${ }^{9}$

One potential solution to this issue is to exploit the concept of splenic switch off. Splenic contraction is not a new phenomenon-it was originally described in 1963 
in animal physiology experiments involving exercise and pharmacological stress. ${ }^{10}$ Over 30 years ago, it was reported in radionuclide ventriculography among patients undertaking maximal symptom-limited supine bicycle ergometer exercise. ${ }^{11}$ It was not until 2015, however, that the CMR imaging community embraced its potential for gauging the adequacy of adenosine vasodilator stress in real-world clinical practice. $^{12}$ Splenic perfusion in vivo is intrinsically regulated by adenosine via the $\mathrm{A} 1$ and $\mathrm{A} 2 \mathrm{~B}$ receptors; endogenous adenosine results in a reduction in splanchnic blood flow via an A1-mediated mechanism and mediates coronary vasodilation by acting predominantly on the $\mathrm{A} 2 \mathrm{~A}$ receptor. ${ }^{13}$ It is worth emphasizing that SSO is not observed in patients undergoing regadenoson stress due to its specificity as a A2A receptor agonist; SSO is, therefore, only applicable to adenosine and dipyridamole.

The study presented by Bakula and colleagues in the current edition of $\mathrm{JNC}$ is a welcome addition to the literature. ${ }^{14}$ The authors should be commended for their efforts to compare the role of adenosine-induced splenic switch off (SSO) as a marker of adenosine stress adequacy in nitrogen-13 ammonia PET versus CMR. The use of simultaneous hybrid PET/CMR multi-modality imaging and the inclusion of both quantitative parameters of splenic activity alongside visual assessment for SSO are important advantages of the study. The demonstration of the feasibility of adopting SSO in PET MPI as well as CMR practice is the study's major finding. Using CMR as the reference standard, the authors used a ROC analysis to demonstrate that a splenic activity ratio (SAR) of 0.46 was the most accurate cut-off for prediction of SSO.

All of the current PET scanners with CT component will allow inclusion of the spleen within the imaging field of view. Indeed, in an observational cohort study of 839 patients in which $703(84 \%)$ of the dipyridamole $\mathrm{Rb}-82$ PET MPI scans included visualization of the spleen, splenic response ratio (equivalent to SAR) was independently associated with major adverse cardiovascular events in subjects without ischemia (defined by a normal summed difference score on relative perfusion imaging). ${ }^{15}$ Thus, as well as identifying patients with inadequate pharmacologic stress and minimizing falsenegative results, splenic response may offer prognostic utility.

The study of Bakula et al. ${ }^{14}$ is, however, subject to some limitations. The decision to include myocarditis patients in this cohort is debateable. Myocarditis is a heterogeneous condition with a wide spectrum of clinical presentations, causes and pathologies; equally, only 14 patients (28\%) included in the study had a history of coronary artery disease at baseline. Using visual assessment of SSO by CMR as the reference standard technique was both practical and logical; however, the inclusion of more patients with coronary imaging (only 2 in this study) would have proven insightful. Finally, despite the recognized drawbacks of using changes in hemodynamic parameters to gauge stress adequacy, because this information is still used in routine clinical practice to help determine stress adequacy, the inclusion of such indices (including rate pressure product) and how these related to the presence/absence of SSO would have improved the study.

In the era of quantitative MPI which is feasible with PET CT (and not yet with dynamic SPECT), SSO may be useful in patients undergoing adenosine/dipyridamole stress to differentiate between inadequacy of stress (SSO absent, very low myocardial perfusion reserve (MPR) and normal relative perfusion) and balanced ischemia/ microvascular dysfunction (SSO present, low or very low MPR and normal relative perfusion; see Figure). As microvascular dysfunction is a diagnosis of exclusion, this would require invasive angiogram or CT coronary angiogram (feasible with hybrid PET/CT systems in a single setting) to confirm absence of obstructive epicardial disease. 


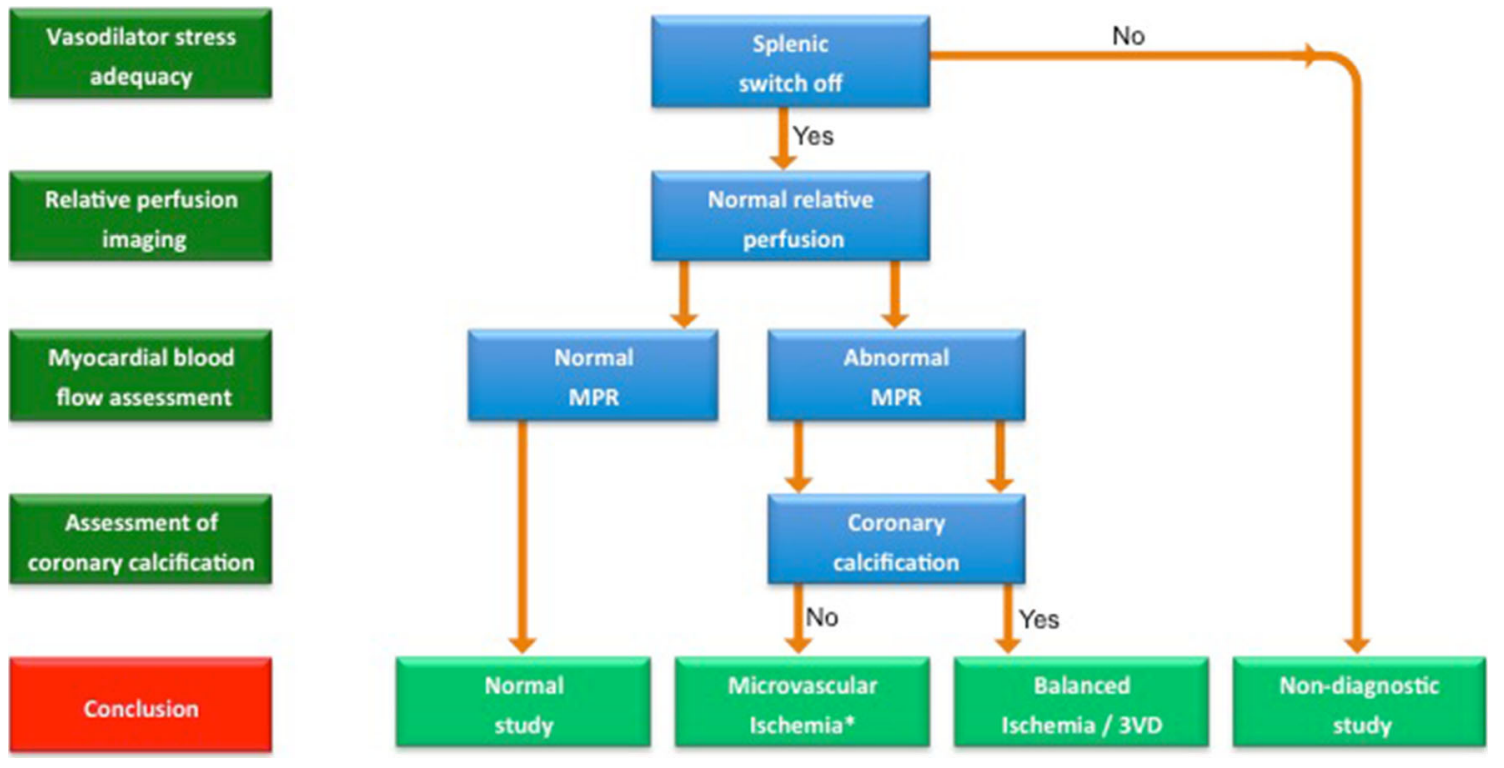

Figure. Proposed algorithm for the inclusion of splenic switch off in a hybrid imaging approach to distinguish between inadequate stress and reduced global myocardial perfusion reserve secondary to balanced ischemia from 3-vessel coronary disease and microvascular dysfunction. MPR Myocardial perfusion reserve; 3VD 3-vessel coronary artery disease. *Microvascular dysfunction is a diagnosis of exclusion, which would require invasive angiogram or CT coronary angiogram (feasible with hybrid PET/CT systems in a single setting).

In summary, this study highlights an important future role for SSO as an imaging tool in the assessment of stress adequacy during adenosine (and dipyridamole) perfusion PET/CT imaging and suggests that this simple observation should not be limited exclusively to CMR imaging units.

\section{References}

1. Tilkemeier P, Green J, Einstein AJ, Fazel R, Reames P, Shaw LJ. The evolving practice of nuclear cardiology: results from the 2011 ASNC member survey. J Nucl Cardiol 2012;19:1170-5.

2. Henzlova MJ, Duvall WL, Einstein AJ, Travin MI, Verberne HJ. ASNC imaging guidelines for SPECT nuclear cardiology procedures: Stress, protocols, and tracers. J Nucl Cardiol. 2016;23:60639.

3. Karamitsos TD, Ntusi NA, Francis JM, Holloway CJ, Myerson SG, Neubauer S. Feasibility and safety of high-dose adenosine perfusion cardiovascular magnetic resonance. J Cardiovasc Magn Reson 2010;12:66.

4. Kidambi A, Sourbron S, Maredia N, Motwani M, Brown JM, Nixon J, et al. Factors associated with false-negative cardiovascular magnetic resonance perfusion studies: A Clinical evaluation of magnetic resonance imaging in coronary artery disease (CEMARC) substudy. J Magn Reson Imaging 2016;43:566-73.
5. Brubaker PH, Kitzman DW. Chronotropic incompetence: causes, consequences, and management. Circulation 2011;123:1010-20.

6. Moody WE, Holloway B, Arumugam P, Gill S, Wahid YS, Boivin $\mathrm{CM}$ et al. Prognostic value of coronary risk factors, exercise capacity and single photon emission computed tomography in liver transplantation candidates: A 5-year follow-up study. J Nucl Cardiol 2020.

7. Bhutani S, Tobis J, Gevorgyan R, Sinha A, Suh W, Honda HM, et al. Accuracy of stress myocardial perfusion imaging to diagnose coronary artery disease in end stage liver disease patients. Am J Cardiol 2013;111:1057-61.

8. Mc Ardle BA, Dowsley TF, deKemp RA, Wells GA, Beanlands RS. Does Rubidium-82 PET have superior accuracy to SPECT perfusion imaging for the diagnosis of obstructive coronary disease? J Am Coll Cardiol 2012;60:1828-37.

9. Tilkemeier PL, Bourque J, Doukky R, Sanghani R, Weinberg RL. ASNC imaging guidelines for nuclear cardiology procedures : Standardized reporting of nuclear cardiology procedures. J Nucl Cardiol 2017;24:2064-128.

10. Guntheroth WG, Mullins GL. Liver and spleen as venous reservoirs. Am J Physiol 1963;204:35-41.

11. Sandler MP, Kronenberg MW, Forman MB, Wolfe OH, Clanton JA, Partain L. Dynamic fluctuations in blood and spleen radioactivity: Splenic contraction and relation to clinical radionuclide volume calculations. J Am Coll Cardiol 1984;3:1205-11.

12. Manisty C RD, Herrey AS et al. . Splenic switch-off: a tool to assess stress adequacy in adenosine perfusion cardiac imaging. Radiology 2015;276(3):732-740. Radiology 2015;276 732-40. 
13. Fozard JRM-KM. Contraction of the rat isolated spleen mediated by adenosine $\mathrm{A} 1$ receptor activation. $\mathrm{Br} \quad \mathrm{J}$ Pharmacol 1993;109:1059-63.

14. Bakula A, Patriki, D., von Felten, E., Benetos, G., Sustar, A. et al. Splenic switch-off as a novel marker for adenosine response in nitrogen-13 ammonia PET myocardial perfusion imaging - crossvalidation against CMR using a hybrid PET/MR device. J Nucl Cardiol 2020.
15. Bami K, MD, Tewari, S., Guirguis, F., Garrard, L., Guo, A. et al. Prognostic utility of splenic response ratio in dipyridamole PET myocardial perfusion imaging. J Nucl Cardiol 2018;26:1888-97.

Publisher's Note Springer Nature remains neutral with regard to jurisdictional claims in published maps and institutional affiliations. 